\title{
Domination sociale et représentations du juste
}

Mise à l'épreuve d'une thèse d'Axel Honneth

\section{Caroline Guibet Lafaye}

\section{OpenEdition}

\section{Journals}

Édition électronique

URL : http://journals.openedition.org/ress/2284

DOI : $10.4000 /$ ress. 2284

ISSN : 1663-4446

Éditeur

Librairie Droz

\section{Édition imprimée}

Date de publication : 1 décembre 2012

Pagination : 147-174

ISBN : 978-2-600-01704-6

ISSN : 0048-8046

\section{Référence électronique}

Caroline Guibet Lafaye, " Domination sociale et représentations du juste », Revue européenne des sciences sociales [En ligne], 50-2 | 2012, mis en ligne le 01 janvier 2016, consulté le 21 avril 2019. URL: http://journals.openedition.org/ress/2284; DOI : 10.4000/ress.2284 


\title{
DOMINATION SOCIALE ET REPRÉSENTATIONS DU JUSTE MISE À L'ÉPREUVE D'UNE THĖSE D'AXEL HONNETH
}

CAROLINE GUIBET LAFAYE

CNRS - Centre Maurice Halbwachs caroline.guibetlafaye@ens.fr

\begin{abstract}
Résumé. La théorie critique d'Axel Honneth décrit la morale sociale des groupes opprimés en termes de «sentiment d'injustice» et récuse le fait qu'elle enferme des représentations abstraites d'un ordre moral général ou d'une société parfaite. La référence à la domination joue, dans cette analyse, un rôle cardinal. Pourtant dans le contexte contemporain, l'identification de «classes dominées» n'a plus rien d'immédiat. Afin d'apprécier la variabilité des représentations et des jugements relatifs au juste et à l'injuste, en fonction des positions sociales, nous envisagerons, dans un premier temps, les limites de cette interprétation dichotomique, lorsqu'il est question de justice sociale. Nous proposerons ensuite une réévaluation, dans le domaine de la critique de la thèse portant sur la cohérence argumentative de la conscience morale commune. Nous montrerons enfin que la projection de registres du discours sur des catégories socialement distinctes ne trouve plus aujourd'hui la pertinence qu'elle a pu antérieurement revêtir.
\end{abstract}

Mots-clés: justice, inégalités, sentiments moraux, domination, Axel Honneth.

\begin{abstract}
Axel Honneth describes the social morality of dominated classes in terms of mere "sense of injustice" and contests that it involves abstract representations of a general moral order or of a perfect society. The reference to domination plays a cardinal role in his analysis. Yet in the contemporary context, the identification of "subordinate classes" has nothing immediate. In order to assess the variability of interpretations and judgments relating to justice and injustice, according to social positions, we will consider, firstly, the limits of the Honnethian dichotomous interpretation, regarding social justice issues. Then about theses issues we propose a reassessment of criticism against the thesis about the argumentative coherence of common moral consciousness. We finally show that the projection of registers of discourse on socially distinct categories is no longer relevant today.
\end{abstract}

Keywords: justice, inequalities, moral feelings, domination, Axel Honneth. 
Héritier de l'école de Francfort, fondée au début des années 1930 par Max Horkheimer et Theodor Adorno, et successeur de Jürgen Habermas à l'université de Francfort, Axel Honneth (1949-) développe, à partir de la fin des années 1990, dans plusieurs textes épars ${ }^{1}$, une analyse des représentations du juste des groupes antagonistes dans l'espace social, en l'occurrence de ceux qu'il désigne comme les dominants et les dominés. Dans l'article sur lequel nous nous appuierons, «Moralbewusstein und soziale Klassenherrschaft» (I98I) du début de sa carrière mais qu’Axel Honneth n’a cessé de faire réimprimer, le soubassement bourdieusien de sa théorie sociale et politique est très net. Le philosophe et sociologue ${ }^{2}$, illustrant parfaitement la tradition allemande des sciences sociales passant outre les frontières séparant philosophie et sociologie, décrit la morale sociale des groupes opprimés en termes de «sentiment d’injustice »-négligés par la théorie habermassienne concentrée sur les règles formelles de la communication - et récuse qu'elle enferme des «représentations abstraites d'un ordre moral général» et légitimé ou encore «d'une société parfaite» (Honneth, 2006, p. 208). Honneth s'appuie alors sur la vision bourdieusienne de jeux de langage normatifs socialement acquis, voire socialement constitués, reflétant les tentatives des groupes dominants de justifier leur position. Cette perspective animera encore ses analyses ultérieures des luttes pour la reconnaissance symbolique, entre des groupes occupant des positions asymétriques, dans la hiérarchie sociale (Den Brink et Owen, 2007 ; Caillé et Lazzerri, 2009), bien que progressivement la question de la domination de classe tende, dans l'œuvre honnethienne, à céder le pas à une interprétation de la conflictualité sociale à partir d'un paradigme communicationnel et psychologique, respectivement inspiré de Jürgen Habermas et de George H. Mead (Ulrich, 2009). A contrario, les auteurs contemporains s'inspirant de la critique

I Une partie des textes élaborés par Axel Honneth entre 1994 et 2004 ont été rassemblés et traduits en français dans le recueil, La Société du mépris (La Découverte, 2006 [2000]). Ils sont thématiquement organisés autour de la théorie critique de l'École de Francfort, de la théorie de la reconnaissance ainsi que des pathologies et paradoxes des sociétés capitalistes contemporaines. Voir infrabibliographie complémentaire.

2 Directeur de l'Institut de recherche sociale à Francfort-sur-le-Main depuis 200I. Sur la tradition de la philosophie critique allemande, voir Rainer, 2009, et sur l'évolution de la théorie critique d'Axel Honneth, voir Iser, 2008. 
sociale honnethienne replaceront au centre de leurs analyses la référence à la domination sociale (voir Renault, 2004; Deranty, 2009).

Dans les années 1980, Honneth estime que les groupes opprimés-à la différence des couches socialement dominantes-ne se rapportent pas à l'équité et à l'injustice en référence à un système de valeurs généralisé, positivement orienté en fonction de normes morales. Honneth s'appuie sur la sociologie des classes opprimées pour analyser les conditions de formulation de leurs conceptions morales, alors ignorées par Habermas. Un regard critique, suggéré par des données empiriques récemment recueillies (voir infra), introduit cependant une double interrogation : la référence à la domination sociale, en matière de représentations et d'interprétations du juste, a-t-elle encore, dans le contexte social français actuel, une pertinence? L'hypothèse, formulée par Axel Honneth, de la coïncidence de représentations d'un monde juste ainsi que de sentiments d’injustice avec des positions sociales spécifiques, a-t-elle toujours une pertinence empirique? En effet, dans le contexte contemporain qui a notamment été celui de l'élargissement des classes moyennes (Mayer, 2003, p. IoI), l'identification de «classes dominées » n’a plus rien d’immédiat, alors qu’elle pouvait sembler évidente dans des sociétés structurées autour de la dualité «monde ouvrier » / «bourgeoisie », auxquelles font référence les travaux empiriques sur lesquels Barrington Moore (1978) et Axel Honneth, à sa suite, s’appuient.

Sans assumer une interprétation de la domination sociale en termes d'opposition de classes ni prétendre trancher le débat sur la persistance des classes sociales, nous envisagerons la notion de domination à partir d'une acceptation minimale de cette dernière: dominants et dominés se distinguent, respectivement, comme «ceux auxquels l'ordre social profite et bénéficie et ceux auxquels il nuit» (Fischbach, 20ı, p. I02). «Le dominant est celui qui occupe dans la structure une position telle que la structure agit en sa faveur» (Bourdieu, 2000, p. 238). Cette approche, sans statuer sur la domination de classe, permet de tenir compte des phénomènes de désavantage social, frappant certains individus et dont témoignent notamment la dégradation des conditions de travail (Linhart, 20 I ; Deranty, 20I I) ainsi que la multiplication des emplois précaires. 
Nous mettrons donc à l'épreuve la thèse d'Axel Honneth concernant la spécificité des représentations du juste des groupes socialement désavantagés, en la confrontant à des données empiriques récentes, recueillies dans le cadre de l'enquête Perception des inégalités et sentiments de justice (PISJ), menée durant l’hiver 20ı0-20ı i dans cinq zones géographiques françaises: les régions de Grenoble, Lille, Lyon, Nantes, Paris. Volet qualitatif, d'une enquête comportant un volet quantitatif, cette recherche a bénéficié d'un financement de l'Académie des sciences morales et politiques (Fondation Simone et Cino del Duca). Les individus interrogés ont été recrutés conformément à un échantillonnage «boule de neige», s'efforçant de respecter une certaine diversité sociologique (voir annexe I). Ces cinquante-et-un entretiens approfondis ont été enregistrés et retranscrits intégralement, pour ensuite être soumis à une analyse classique de contenu ainsi qu'à une analyse textuelle à l'aide du logiciel Alceste.

Afin de répondre à la question de la variabilité des représentations et des jugements relatifs au juste, en fonction des positions dans l'espace social, nous envisagerons, dans un premier temps, les limites de cette interprétation dichotomique dans le domaine des représentations de l'injustice et des inégalités. Nous proposerons ensuite une réévaluation de la critique de la thèse défendant la cohérence argumentative de la conscience morale commune, en matière de justice sociale. Nous montrerons, pour finir, que la projection de registres du discours, tels que la justification d'états sociaux, par opposition à leur dénonciation, sur des catégories socialement distinctes ne trouve plus aujourd'hui la pertinence qu'elle a pu antérieurement revêtir.

\section{REPRÉSENTATIONS MORALES ABSTRAITES VS. SENTIMENTS D'INJUSTICE TYPIQUES}

\section{I.I. CONSCIENCE MORALE ET DOMINATION DE CLASSE}

Dans l'article « Moralbewusstein und soziale Klassenherrschaft », Axel Honneth établit une dichotomie stricte entre deux types de rapport à la justice sociale. Sa thèse centrale [A] réside dans l'affirmation selon laquelle les conceptions de la justice des couches opprimées seraient «implicitement contenues dans des sentiments 
d'injustice typiques » plutôt qu'elles ne se traduiraient dans des principes axiologiques explicites, à la différence de ce dont témoigneraient les représentations morales abstraites des groupes dominants (Honneth, 2006, p. 212). Or cette thèse comporte au moins trois implications distinctes.

En premier lieu et bien que les sentiments d'injustice, exprimés par les classes dominées, enferment en creux «un potentiel d'exigences de justice, de besoins et d'idées du bonheur» (idem), (I) les critères implicites de réprobation morale qui sont mobilisés par ces groupes, dans l'expression de sentiments d'injustice, ne sont pas, selon Honneth, «organisés en un système distancié de normes abstraites » ni ne se cristallisent en projets de société juste. Ce phénomène ne tient pas à une infériorité cognitive des classes sociales concernées mais au fait que la question normative exerce sur celles-ci une pression directe moindre. Ce faisant, la morale sociale de ces groupes exprimerait plutôt, selon l'auteur, «une sensibilité exacerbée aux atteintes contre des revendications morales jugées légitimes». Elle signalerait seulement, «avec la force de l'expérience vécue, les possibilités de justice écartées par la morale hégémonique» (ibid. p. 209).

Ainsi - et telle est la deuxième implication de la thèse honnethienne - (2) la «moralité intérieure », déposée dans l'ensemble des critères qui nourrissent les condamnations, exprimées par les groupes opprimés, ne serait que le négatif d'un ordre moral institutionnalisé. Interrogés sur les principes axiologiques susceptibles de fonder un ordre social, les individus subissant la domination se cantonneraient systématiquement dans des «stéréotypes normatifs » (ibid., p. 2I I). (3) Engluées dans la colère et l'indignation, les classes dominées s'en tiendraient à «un ensemble disparate de revendications réactives». Cette troisième implication de la position honnethienne, initialement mentionnée, suggère que les prémisses axiologiques mobilisées par ces groupes ainsi que leurs conceptions de la justice demeurent inapparentes, leur système de valeurs explicite étant souvent jugé conventionnel par Axel Honneth.

L'interprétation, proposée par l'instigateur de la théorie de la reconnaissance, institue une asymétrie caractérisée, de nature à la fois représentationnelle, expressive et normative entre groupes sociaux antagonistes. Elle souligne, comme sa conséquence, l'existence d'une domination normative de classe. D’une 
part, les sentiments d'injustice des classes dominées, ne pouvant «s'appuyer sur un système alternatif du même degré d’abstraction que celui des classes dominantes, s'en tien[draien]t aux normes hégémoniques, sans pourtant entériner leur prétention à la validité sur un plan normatif» (ibid., p. 217). D’autre part et dans la mesure où les individus des couches opprimées demeureraient en deçà de la formulation explicite de principes axiologiques, lorsqu'ils évaluent et jugent l'ordre social sur le plan moral, les critères de la réprobation morale convoqués - pour apprécier certains processus sociaux - doivent inévitablement être cherchés dans des systèmes axiologiques, développés dans d’autres sphères sociales.

Cet ensemble de thèses se justifie, dans la perspective honnethienne, par le contexte social auquel il fait référence. Les conditions socio-structurelles sont instituées en facteurs explicatifs des orientations morales et des dispositions normatives des groupes opprimés, lesquelles échapperaient à « toute exigence de cohérence [normative], qu'elle soit institutionnelle ou informelle» (ibid., p. 209). Leur cadre professionnel et social ne contraint aucunement ces groupes à produire un système de valeurs cohérent et légitimant. Le climat culturel des classes opprimées, dans cette lecture sociohistorique, ne les pousserait donc pas à donner à leurs convictions normatives une forme élaborée. Ces raisons socio-structurelles expliqueraient que les exigences de justice de ces groupes ne s'expriment que dans les diverses formes du sentiment d'injustice (ibid., p. 212).

Bien que ce cadre interprétatif ait pu avoir une pertinence sociale dans la seconde moitié du Xxe siècle, en particulier jusquaux années 1980, il demande à être réévalué à la lumière des évolutions sociales contemporaines. Plusieurs questions se posent: dans quelle mesure peut-on encore aujourd'hui projeter sur des groupes sociaux des attitudes différenciées à l'égard de la normativité et de l'abstraction? Les groupes sociaux les moins avantagés socialement ne déploient-ils aucune représentation abstraite du juste? Les résultats de l'enquête PISJ, menée en 20I0-20I I, viennent à l'appui de la récusation des thèses d'Axel Honneth ici rappelées. Cependant les limites du modèle honnethien ne sont pas simplement historiques mais également normatives car il repose sur une théorie du jugement moral, relatif à la justice sociale, ainsi que sur une analyse de l'expression des sentiments d'injustice partielles. 


\section{I.2. ABSTRACTION DES SYSTÈMES NORMATIFS VS. SENTIMENTS D'INJUSTICE TYPIQUES}

La première proposition honnethienne, que l'enquête menée permet de récuser, concerne le fait que les individus occupant des positions sociales désavantageuses n'ont pas de représentation abstraite de l'ordre social - à la différence des individus les plus avantagés socialement - mais s'en tiennent à l'expression brute de sentiments d'injustice. Concernant ces groupes, Axel Honneth souligne que «les exigences empiriquement opérantes ne prennent souvent $[\ldots]$ que la forme d'un sentiment d'injustice relativement persistant, proche de l'expérience immédiate, basé sur des idées de justice inexprimées et non accordées entre elles » (ibid., p. 216-217).

Les cinquante-et-un entretiens PISJ permettent d'identifier vingt-neuf individus dont les positions dans le monde professionnel sont dominées et quatre enquêtés (des femmes) dont la situation de domination repose sur des facteurs non exclusivement professionnels. Ces quatre femmes appartiennent à la classe moyenne mais le confort de leur situation tient à leur statut conjugal et aux revenus de leur conjoint ${ }^{3}$. Les traits qu'Axel Honneth estime caractéristiques du discours des dominés, en l'occurrence une faible explicitation des principes de justice convoqués dans le discours évaluatif, l'accumulation de stéréotypes normatifs, la prévalence des sentiments d'injustice sur l'expression de représentations abstraites et cohérentes du juste - c'est-à-dire les trois thèses précédemment décrites -, ne s'affirment que parmi un nombre quantitativement très restreint d'individus (cinq sur vingt-neuf) [groupe $\alpha, n=5]^{4}$. La nature de leurs discours ne semble en outre pas tant s'expliquer par les structures de la domination sociale que par un faible niveau de diplôme puisqu'un seul individu parmi eux peut se prévaloir d’un niveau de diplôme supérieur au baccalauréat.

3 Lorsqu'elles ont un revenu, celui-ci est compris entre 1200 et $1700 €$.

4 Est placée en annexe 2 une partie des questions de la grille d'entretien qui a permis de reconstruire ces représentations. 
À l'inverse, les représentations morales, les conceptions de la justice et les dispositions cognitives de plus des deux tiers des individus, dotés de situations professionnellement dominées [groupe $\beta, \mathrm{n}=\mathrm{II}$ ], ébranlent la description honnethienne. Ces individus déploient un discours construit, promouvant un système normatif alternatif à celui prôné par l'idéologie dominante et ne présentant aucune trace d'intériorisation de cette dernière. Ces enquêtés se montrent attachés à la promotion des conceptions individuelles du bien dans leur diversité, d’autres à une société plus intégrée et socialement plus mixte, plus soucieuse d'écologie et de développement durable, plus solidaire voire fondée sur le principe de différence (voir infra l'extrait de l'entretien avec Dominique) et le droit à la vie reconnu et garanti à tous. Ces enquêtés, plus diplômés que ceux du groupe précédent (c'est-à-dire du groupe $\alpha$ ), ont en commun d'être tous Français de parents d'origine française. S’associe à ce profil, le groupe des quatre femmes précédemment décrit, portant ainsi leur nombre à quinze.

Entre ces deux groupes contrastés, se dresse l'ensemble des individus, professionnellement désavantagés ${ }^{5}$, dont les discours présentent des degrés variables d'élaboration de conceptions abstraites de la justice, associées à des formes de légitimation partielle de la domination [groupe $\gamma, \mathrm{n}=\mathrm{I3}$ ]. La moitié environ d'entre eux $[\mathrm{n}=7]$ endosse une représentation abstraite d’une société juste, incluant une légitimation partielle des phénomènes de domination. Chez à peine un quart $[\mathrm{n}=3]$, le discours de la légitimation est plus accentué. Dans le dernier quart $[\mathrm{n}=3]$, en revanche, l'expression de l'injustice et de la révolte se fait plus virulente, tout en sassociant - voire en se justifiant - d'une représentation abstraite de la justice ${ }^{6}$.

5 Cet ensemble concerne des statuts hétérogènes excluant toutefois les cadres supérieurs du privé et les professions libérales. II rassemble, d'une part, des professions soumises à des conditions de travail défavorables (temps partiel subi, statut d'ouvrier placé dans des conditions d'exercice difficile), d'autre part, des statuts professionnels socialement dévalorisés (travailleurs sociaux s'occupant de populations très marginalisées) ou faiblement reconnus (intermittent du spectacle), ou encore des situations de désajustement professionnel, des professions très faiblement rémunérées et des situations de chômage. Aucun de ces individus n'a des revenus mensuels supérieurs à $1700 €$ si ce n'est Thibault dont le revenu se situe entre 1700 et $2400 €$ mais dont le contexte de travail présente des contraintes très fortes.

6 Les trois individus de ce dernier sous-groupe ont en commun d'être issu de l'immigration. L'enquête par questionnaire suggère que la comparaison du «sentiment d'être défavorisé », dans divers registres socio-économiques, exprimé par les Français d'origine française et les Français nés de parents étrangers, manifeste une différence en défaveur des seconds, concer- 
Qu'ils développent un discours construit et articulé à des principes moraux, qu'ils se gardent d'exprimer brutalement des sentiments d'injustice pour privilégier un discours descriptif, ou qu'ils se cantonnent dans la résignation et la fatalité, le discours de ces individus récuse les interprétations honnethiennes du rapport cognitif et moral au juste et à l’injuste des groupes socialement dominés.

Seuls quelques enquêtés, plutôt rares, expriment leur révolte et leur indignation avec virulence. L'intensité de ces sentiments ne relègue pourtant pas le discours qui les porte dans «la forme d'un sentiment d'injustice relativement persistant, proche de l'expérience immédiate, basé sur des idées de justice inexprimées et non accordées entre elles » (Honneth, 2006, p. 217). Des principes de justice et des convictions morales motivent et justifient, à leurs yeux et pour autrui, leurs sentiments d'injustice. Évoquons le discours de François, travailleur social, relatif aux inégalités en santé :

L’inégalité, je crois que c'est la première là, l'accès aux soins [...]. Moi j’en parle parce que je suis directement concerné dans l'asso où je bosse [...]. Toutes les personnes qui bénéficient de la CMU et qui se font jeter par les médecins, qui ont pas les moyens de se payer leurs médicaments, tout simplement [...]. Ceux qui touchent la CMU en fait normalement, ils ne doivent rien payer, mais ils peuvent pas se soigner parce que les médecins ils refusent.

Les entretiens menés suggèrent en outre qu'à l'exception d'une occurrence unique, les individus les moins diplômés n'éprouvent pas de sentiment d'injustice, pour des situations les concernant personnellement, ou sont les moins enclins à considérer avoir été victimes d’injustice. Ainsi Thibault, ouvrier qualifié, avoue : «Toujours dans l'enfance, je pense qu'on a toujours l'impression d'être traité injustement par ses parents, des choses comme ça, mais de là à dire que c'était une injustice totale, c'est un peu gros comme mot». 
Les récits rassemblés permettent certes, pour une autre part, de vérifier que les individus bénéficiant de positions socialement favorables expriment, assez largement, des représentations abstraites de la société juste et procèdent fréquemment à une explicitation des principes de justice qui sous-tendent leur conception d'une société juste. Cependant, si une «représentation abstraite de l'ordre social» signifie, comme le suggère Honneth (ibid., p. 2II), la «projection d'une société parfaite», il ne fait pas de doute que les enquêtés moins avantagés ont une représentation - en général assez nette - de ce que serait cette «société parfaite» ou juste. De ce point de vue, le discours de Dominique, aujourd'hui au chômage, est évocateur :

Pour moi c'est à ça qu'on mesure le degré d'humanité, de civilité [...] d'une société, c'est-à-dire qu'on ne laisse personne derrière [...] s'il y a un effort à faire c'est de ce côté-là, ce n'est pas dans le fait de rémunérer mieux ceux qui gagnent déjà largement de quoi vivre correctement, non, c'est d'amener tout le monde à avoir un niveau de vie qui soit acceptable.

Se dessinent donc également, du côté des individus les moins avantagés socialement, des «systèmes de valeurs englobants » et des conceptions explicites de la justice sur lesquelles nous reviendrons ultérieurement.

Ces conceptions du juste n'émanent certes pas de tous les récits des individus socialement désavantagés. Dans cette mesure, la référence à la domination sociale n'est peut-être pas le facteur explicatif ultime de ces différences de représentations. Ainsi que nous l'évoquions en introduction, la question fondamentale est celle de savoir si l'inadéquation des thèses d’Axel Honneth tient à la référence à des structures de domination, i.e. à l'existence de phénomènes de domination sociale, ou au fait de projeter des représentations du juste ainsi que des structures du langage sur des catégories sociales. Comme nous l’avons souligné, l’absence de systèmes de valeurs généralisés ou de principes axiologiques sous-tendant le jugement moral n'est patente que dans le discours d'une minorité d'individus, subissant des formes de domination professionnelle, qu'ils soient ouvriers ou intermittents du spectacle (voir Menger, 2005). Bien quappert, dans le discours de personnes vivant des situations professionnellement dominées, une tendance à ancrer leurs explications de l'état du monde «dans un principe unique» (qu'il s'agisse de l'argent, de la reproduc- 
tion sociale, de la domination ou du respect des choix de vie individuels) ${ }^{7}$, les récits de la plupart de ces individus convoquent des référentiels axiologiques très précis et invitent à nuancer les conclusions d'Axel Honneth.

La nature et les formes prises par les discours de ces personnes s'expliquent avec plus de pertinence par leur niveau d'éducation plutôt que par le fait d’assumer des situations désavantagées, de hauts niveaux de diplôme ne garantissant pas toujours des situations professionnelles enviables. D’autres facteurs, tels qu'un accès plus large à l'information, une augmentation du niveau moyen d'éducation de la population ou la participation à des structures associatives de type syndical, peuvent expliquer que nombre de ces individus mobilisent des systèmes axiologiques précis. Un niveau de diplôme inférieur, associé à des professions d'exécution, n'est en outre pas un obstacle systématique à la formulation de représentations abstraites de la justice, comme le récit de Jannick, ouvrier qualifié syndiqué de longue date, le montre ${ }^{8}$. Même des ouvriers faiblement diplômés déploient un discours critique construit sur le fonctionnement macrosocial (capitaliste et financier) ou concernant les méthodes contemporaines de management des grandes entreprises 9 . Très rares - l'occurrence unique est donnée par Henry - sont les enquêtés dont le discours n'illustre qu'«un ensemble disparate de revendications réactives ».

7 Ainsi Max, artisan retraité, suggère que «c'est pas le chef d'État qui dirige, c'est les banquiers, l'État peut essayer [...] d'influencer certaines choses, d'ailleurs y a qu'à regarder ce qui se passe avec les banques et tout ça. C'est les financiers qui commandent, c'est l'argent qui commande $[\ldots]$ ». François, comme Daniel, journaliste, estime que «la société est pourrie, que c'est l'argent qui commande tout».

8 Yannick considère par exemple que la société française est «complètement [hiérarchisée]. Et ça ne date pas d'aujourd'hui, ça date du début des temps. Et pas qu'en France, partout. [...] C'est comme les salaires, c'est pareil, c'est hiérarchisé, c'est une échelle. À l'époque c'était de un à 32, dans les années 1970. Maintenant [...] ça doit être sensiblement la même chose».

9 Jean-Yves, ouvrier, souligne que «Nord Pack - il fait partie de Bouygues [...] Moi je travaille sur Valenciennes et du jour au lendemain, ils ont payé le ler mai et le 8 mai, [...] ils n'avaient plus de travail, soit disant [...]. Ils nous ont renvoyés pour pas payer la journée du 8 mai qui était fériée [...]. La semaine d'après, ils nous ont recontactés pour revenir travailler [...]. On est rejeté... et ça c'est des choses... que j'ai vécues [...] du jour au lendemain, qu'ils nous remettaient sur d'autres chantiers tout ça, non [...] Pour pas payer le 8 mai, ils ont dit "non, y'a plus de travail, on a plus besoin de vous..." ». 
De même, à l'encontre de la thèse honnethienne (I) selon laquelle les groupes dominés ne portent pas de représentations abstraites d'une société juste, les entretiens menés suggèrent que les situations de domination professionnelle actuelles n'entravent pas systématiquement l'expression normative du juste ni n'interdisent, chez ceux qu'elles touchent, toutes «projections d'une société parfaite», notamment parce qu'elles ne sont pas exclusives de niveaux de diplôme relativement élevés ou supérieurs à la moyenne. Ainsi Charlotte, assistante sociale à temps partiel, note :

J'crois pas à une société où on serait tous, genre le niveau de vie... L'égalité pour moi elle existe pas, j’veux dire, on est tous différents, avec un bagage différent, avec un vécu différent [...]. C’est inévitable, [...] on peut pas tous vivre de la même manière puis on a pas tous envie de vivre de la même manière aussi quoi [...]. À partir du moment où les envies de chacun sont respectées et que l'intégrité de la personne est respectée, ça me paraît... c'est surtout ça qui me paraît important.

Cette conjonction s'observe notamment pour des personnes assumant des professions du social. Du fait de l'extension des situations de précarité professionnelle à des individus jeunes et fortement diplômés (de niveau bac + 3) - c'est-à-dire plus diplômés que les ouvriers en référence auxquels Axel Honneth a construit son discours -, les phénomènes de domination dans le monde du travail n'obèrent pas l'élaboration discursive des expériences de l'iniquité - l'éducation exposant à davantage d'informations -, ni l'expression de sentiments d'injustice cohérents, pas plus qu'ils n'enjoignent à systématiquement endosser des stéréotypes normatifs concernant l'ordre social. Ainsi Jacques, infographiste au chômage, dénonce le mode de fonctionnement des entreprises tournées vers la production plutôt que vers la qualité du travail:

[...] même les boîtes qui font des bénéfices arrivent à faire des plans sociaux. Quand on dit "variable d'ajustement", le mot est bien choisi. Ce n'est pas l'objet de bien travailler. L'objet n'est plus de bien travailler, c'est de produire quand il faut produire, de débaucher quand il faut débaucher.

Des parcours de mobilité sociale descendante sont également à l'origine de situations socialement dominées. Ils concernent des individus ayant bénéficié de niveau d'études avancé et / ou de positions professionnelles jadis avantageuses, les disposant à forger des représentations du monde abstraites et cohérentes. 
On ne peut donc conclure, de façon générale comme le voudrait la première implication de la thèse rappelée d'Axel Honneth, que les conceptions de la justice des couches opprimées sont «implicitement contenues dans des sentiments d'injustice typiques » plutôt qu'elles ne sont formulées dans des principes axiologiques explicites (Honneth, 2006, p. 2г2). Lassignation honnethienne des groupes dominés à l'expression brute de sentiments d'injustice se voit récusée par les données empiriques. Les sentiments d'injustice qu'ils expriment ne constituent pas une médiation systématique de la formulation de principes de justice. En particulier, concernant la santé et le logement, les sentiments d’injustice exprimés sont motivés par le constat du non respect du principe de la satisfaction des besoins fondamentaux, par l'exigence d'un accès universel aux soins de santé ainsi que par le droit égal à la vie. Ainsi Marine, élève aide-soignante, tient ferme que «normalement la santé, elle doit être gratuite pour tous», «tout le monde doit être soigné pareil». Les jugements et les sentiments d’injustice recueillis sont fondés sur des principes de justice et des références normatives, mobilisés par les classes dominées, dans la description de leurs expériences directes ou indirectes d'injustice, au-delà de la seule expression «c'est pas normal! ». Ils se nourrissent de conceptions sous-jacentes d'une société juste, servant de point d'appui normatif à la critique sociale et à la dénonciation de l'injustice.

De surcroît et conformément à la troisième implication de la thèse honnethienne (3), tout de même que le substrat cognitif des dominés ne s'épuise pas dans un «sentiment d'injustice», l'expression de ce type de sentiment ne déserte pas le discours des bénéficiaires du système social. Bien qu’ils soient moins exposés personnellement à l'injustice, ces derniers expriment leur indignation et des formes de réprobation morale, sagissant de configurations qui ne les concernent pas personnellement mais qu’ils jugent néanmoins scandaleuses. Ils concernent, en particulier, les attitudes de passager clandestin ${ }^{10}$, la frustration relative et la paresse, mais aussi le traitement des migrants en France ou des sans-abri (SDF), la grande pauvreté, l'inégalité des chances pour des raisons indépendantes de l'individu qu’elle pénalise. Ainsi Gaëlle, retraitée ayant un niveau de vie confortable, estime quau Canada, 
[...] ils savaient juguler l'immigration; est-ce que c'est ce que voulait faire le gouvernement [de Nicolas Sarkozy], [...] à mon avis il s'y prend mal, il met dehors les gens, est-ce que ce n'est pas à la louche, est-ce que c'est justifié, [...] moi ça me choque, peut-être que c'est ça la solution, les mettre dans l'avion, mais pour ces pauvres, il y en a qui travaillent là depuis un certain temps, pourquoi ils travaillent là, ils ont eu un permis de travail, ils ont eu l'acceptation de leurs patrons, donc, dans la mesure où ils travaillent là et que leurs enfants sont scolarisés, enfin il ne faut pas les jeter quoi, enfin, bon, ça ce n’est pas juste.

Enfin et à l'encontre de la troisième implication de la thèse honnethienne (3), nous avons montré que la capacité à évaluer les faits sociaux dans un système de référence cohérent, en déployant un discours logique et articulé, se retrouve dans l'ensemble de l'espace social. Ainsi l'analyse des récits recueillis récuse la réification essentialiste, projetée sur des groupes sociaux, d'un phénomène qui relève davantage de structures du langage, opposant l'inexplicitation et l'explicitation. Le rapport d'exclusion entre sentiment d'injustice et expression construite, cohérente et logique de conceptions du juste caractérise non pas des groupes sociaux ou des modes d'expression propres à ces derniers - comme le voudrait Axel Honneth - mais des formes irréductibles du discours, des classes d'énoncés typiques, opposant d'un côté la dénonciation brute de faits et, de l'autre, l'évaluation de faits sociaux, dans un système de référence cohérent, indépendamment du lieu depuis lequel ces discours sont prononcés.

L’analyse linguistique évite la réification et la projection sur des catégories sociales d'énoncés typiques. La réprobation et la dénonciation peuvent en effet s'exprimer soit de façon non discursive soit en référence à des principes axiologiques et à des critères normatifs. Les récits d'individus ayant des positions socialement confortables montrent également que, selon que l’on justifie ou dénonce les inégalités ou les injustices, la forme même du discours varie. La justification appelle par elle-même et de façon constitutive l'argumentation, alors que le sentiment d'injustice constitue d’abord et de façon également constitutive l'expression d'une indignation, se formulant de façon réfléchissante et qui peut - ou non -, dans un second temps, se justifier. Dans la mesure où résonne une «atteinte à des idées intuitivement données de la justice », l'indignation, en tant quaffect, fait appel - comme dans un jugement réfléchissant (voir Kant, I990 [I790]) - à 
l'intuition, plutôt qu'à l'entendement, et à une sensibilité, supposée partagée par le locuteur et l'interlocuteur, à la différence du discours de la légitimation ou de la justification. Ainsi Hicham, statisticien du Pôle Emploi, est indigné par l'indifférence parisienne face à des situations de pauvreté extrêmes et s'exclame: «on les [les SDF] retrouve de plus en plus nombreux d'une année sur l'autre. C’est effarant! [...] ce qui est terrible c'est que ça étonne plus personne, on est capable d'enjamber les gens ». En somme, la structure du discours et l’appréciation de l'injustice qu'Honneth projette sur deux groupes distincts de la société (les dominants vs. les dominés) constitue une propriété analytique du discours, selon qu'il se déploie de façon argumentative ou qu'il exprime un sentiment.

Quoiqu'on ne puisse cerner des modalités d'expression du juste caractéristiques de groupes sociaux antagonistes, peut-on en revanche identifier un sens particulier de la justice, partagé par les individus ne bénéficiant pas aujourd'hui des structures sociales? Leurs discours témoignent-ils d'un attachement à des principes de justice spécifiques que ne partageraient pas les bénéficiaires de ce système? Dans ce qui suit, nous explorerons la validité empirique contemporaine de la deuxième implication de la thèse défendue par Axel Honneth, tendant à associer des représentations et des interprétations spécifiques du juste à des positions sociales singulières, s'opposant en termes de domination sociale.

\section{I.3. SENTIMENTS OU CONCEPTIONS DE LA JUSTICE TYPIQUES?}

Y a-t-il, dans les groupes dominés, des «sentiments d'injustice typiques» (Honneth, 2006, p. 212) ou des conceptions de la justice typiques? L’analyse systématique des sentiments d’injustice exprimés au cours de ces récits (Guibet Lafaye, 20I2) montre qu'il n'y a pas de sentiments d'injustice typiques des individus, ne bénéficiant pas des structures sociales contemporaines, mais que certaines situations de désavantage social sont à l'origine de sentiments d'injustice caractérisés ${ }^{11}$. Ces sentiments s'expriment, par exemple, lorsque les individus sont pris dans un système qui tend à l'exploitation, réduit les chances s'offrant aux individus, abuse de positions sociales défavorisées ou asymétriques ou encore lorsque

II Ainsi ce qu'Axel Honneth qualifie de suggestions et/ou de réflexions «spéculatives》 (2006, p. 206 et 223) se voit infirmé par des données contemporaines. 
point le sentiment d'être pris dans une machine dont on subit le fonctionnement ou un fonctionnement perçu comme arbitraire et sur lequel on n'a pas de prise.

La «morale sociale non écrite», identifiée par Honneth comme propre aux dominés et se formulant comme le négatif de l'ordre moral institutionnalisé, ne consiste pas exclusivement, dans les entretiens aujourd'hui réalisés auprès de contemporains, à condamner des situations de fait. S'il est vrai que les sentiments d'injustice, exprimés par les individus subissant des situations de domination, permettent de saisir, en creux, des attentes concernant ce que serait un ordre social juste et bon, ce trait décrit une caractéristique du sentiment d'injustice en tant que tel plutôt qu'un phénomène de classe ainsi que le dévoilent les récits recueillis auprès des individus les plus avantagés socialement comme de ceux qui le sont moins, également du fait que tous, en France, ne puissent être soignés.

Ce qu'Axel Honneth attribue à des groupes sociaux doit plutôt être porté au compte de structures de langage et de l'expression de sentiments d'injustice. Cette convergence de la structure même du sentiment d'injustice et de certaines formes de réprobation morale se laisse en particulier saisir sur la question des personnes privées de logement ou ne pouvant accéder à des soins de santé. Elle suggère un sens commun du juste partagé, au-delà de l'hétérogénéité des positions occupées dans l'espace social.

L’analyse des discours recueillis permet également de mettre en évidence une homogénéité des attitudes, concernant ce que serait un ordre social juste et bon, par delà les clivages sociaux. Tel est le cas, par exemple, de l'école et de ses inégalités. Celle-là, davantage que le monde professionnel, est le lieu où les individus les plus avantagés socialement font, indirectement, l'expérience de l'injustice et se voient confrontés aux inégalités, en particulier à l'inégalité des chances d'enfants de milieux sociaux variés. Or aucun des individus interrogés, parmi les plus avantagés, ne légitime l'inégalité des chances scolaires ni le fait que les différences sociales existantes dans le milieu scolaire soient à la faveur des plus favorisés. La diversité notable d'opinions concernant la légitimité des inégalités de revenus ou de patrimoine, de la part des individus les plus favorisés socialement, se restreint drastiquement, lorsqu'il est question de la «sphère de l'éducation». 
Les sentiments d'injustice, exprimés par les individus les moins avantagés, ne sont donc pas typiques de leurs positions sociales, contrairement à ce que suggère Axel Honneth. Bien que ses thèses, comme telles, ne reçoivent pas aujourd'hui de confirmation empirique forte, une version atténuée de ces dernières trouverait-elle un écho empirique dans les récits recueillis? Autrement dit, peut-on montrer que ces individus défendent des principes de justice spécifiques - caractéristiques de leurs positions - et que leurs conceptions du juste ne consistent qu'en une réponse cognitive à l'injustice (conformément à la thèse (2) d'Axel Honneth) ?

\section{COHÉRENCE DES REPRÉSENTATIONS MORALES INDIVIDUELLES ET PRINCIPES DE JUSTICE DES GROUPES SUBALTERNES}

\section{I. REPRÉSENTATIONS ABSTRAITES D'UN MONDE JUSTE}

Nous avons précédemment souligné l'existence de «systèmes de valeurs généralisés, positivement orientés en fonction de normes morales » (Honneth, 2006, p. 21 I), dans le discours d'individus désavantagés socialement. Ils autorisent une récusation de la thèse, défendue par Axel Honneth, selon laquelle les «appréciations négatives [de ces groupes ne sont] pas généralisées en un système positif de principes de justice» (ibid., p. 208). Cette thèse écartée, il faut encore s'assurer que leurs principes de justice ne se résument pas exclusivement, comme le voudrait Honneth, dans les «possibilités de justice écartées par la morale hégémonique ».

D’une part, le discours des individus interrogés, les plus avantagés socialement, tend généralement et incontestablement à proposer, comme le souligne Honneth, des «conceptions juridiques relativement cohérentes et logiquement ordonnées », des «critères $[\ldots]$ de réprobation morale $[\ldots]$ organisés en un système distancié de normes abstraites ». Néanmoins ces traits ne sont pas absents des narrations des individus assumant des positions moins avantagées puisque les récits de deux tiers d'entre eux récusent cette thèse (voir supra I.2). 
D’autre part, pour des raisons structurelles et du fait de leur position sociale, la «distanciation» constitue nécessairement davantage l’apanage des bénéficiaires du système. Néanmoins des sentiments d'injustice s'expriment également au sein de ce groupe. Sarah, retraitée issue d'un milieu social aisé, est scandalisée par le fait quaujourd'hui le pouvoir soit à l'argent, que l’argent organise et domine la société : «c'est pour ça que je vous ai dit que [...] l'inégalité, c'est l'argent, c'est ça qui est la seule hiérarchie aujourd'hui, et ça je trouve ça épouvantable». Rares sont les individus occupant des positions dominantes qui se montrent indifférents aux injustices et aux inégalités. En revanche, les individus qui ne bénéficient pas de la structure sociale vivent des expériences de domination. L'expression de la révolte et de l'indignation émergera donc logiquement davantage de leurs discours.

Cependant les expériences d'injustice, vécues personnellement et subies, ne sont pas la matrice exclusive de l'expression des sentiments d'injustice. L'analyse systématique de l'articulation entre sentiments d'injustice et conceptions individuelles de la justice (Guibet Lafaye, 2012) met en évidence la préséance de certains critères à partir desquels ces condamnations sont formulées. Se dessine alors un sens a priori de la justice, bâti autour des exigences de satisfaction des besoins fondamentaux, de la reconnaissance de l'utilité sociale des professions, de l'égalité des chances, de la rémunération prioritaire du nombre d'heures travaillées, et à partir duquel les individus formulent et justifient - ne serait-ce que pour eux-mêmes - leurs jugements relatifs au juste et à l'injuste, sans que ce sens du juste ne soit assignable à une catégorie sociale spécifiquement identifiable, au sein des rapports de domination sociale.

En outre, les récits recueillis de ce groupe d'individus suggèrent des formes alternatives d'organisation sociale. Leurs conceptions du monde sont fondées sur des principes axiologiques propres, tels que l'épanouissement de soi ou l'égalité, l'utilité sociale ou la reconnaissance de la pénibilité du travail, le principe d'égalité des salaires (résumé dans l'adage «à travail égal, salaire égal»), le principe d'égale reconnaissance des individus, l'absence de justification possible des inégalités. Pour Charlotte, par exemple, les différences de niveaux de vie sont acceptables, «tolérables» si le principe de dignité est respecté, si «chacun vit dignement». Elle ajoute: «À partir du moment où les envies de 
chacun sont respectées et que l'intégrité de la personne est respectée [...] c'est surtout ça qui me paraît important».

Sont mentionnés, dans les récits de ce groupe d'individus, des modèles sociaux fondés sur des valeurs humaines ou dont l'argent et la finance seraient absents ainsi que des structures sociales dans lesquelles le travail ne serait pas le cour de l'activité individuelle, dans lesquelles seraient garantis à chacun les moyens de réaliser sa conception de la vie bonne ou, au moins, les conditions d'une vie digne, comme le suggère Raïssa, professeur des écoles : «La suffisance et la décence, c'est que tout le monde puisse avoir le minimum qui paraît normal à tout le monde ». Ces récits laissent encore apparaître des conceptions d'une société juste où les cloisonnements sociaux seraient moins prégnants et pèseraient moins sur les destins individuels, où chacun aurait accès à la culture. D’autres enquêtés privilégient une société présentant davantage de mixité et de tolérance. Les hommes interrogés sont soucieux de l'accomplissement ou de la réalisation de $\operatorname{soi}^{12}$, alors que les femmes estiment que les valeurs humaines, le respect de la dignité individuelle, une attention au care, en l'occurrence l'attention à autrui et aux plus fragiles, devraient constituer des principes fondamentaux de l'organisation sociale. Le rôle cardinal, dans la structure de base de la société, du principe de différence, est fréquemment souligné à travers l'idée de «ne laisser personne sur le bas côté». Il actualise le principe matériel de progrès (voir Kant, I986 [1795] et Pogge, 1988), suggérant que l'on vivrait mieux si le progrès général de l'humanité ne laissait personne de côté et si tous y trouvaient son compte.

Les conceptions de la justice les plus élaborées de ce groupe dévoilent donc des interprétations argumentées et cohérentes du système social pris dans son ensemble. Elles convoquent des normes morales à partir desquelles son fonctionnement est appréhendé et suggèrent que les phénomènes d’asymétrie sociale,

12 Ainsi Jacques souligne qu'« on veut impliquer les gens dans le résultat au lieu d'axer sur le développement personnel en ayant une politique [...] on est avec ce terreau humain, on fait des choses pour que les gens soient mieux. Les gens auront plus de rendement. [...] C'est vrai que dans une entreprise rêvée où les gens ont l'occasion d'avoir un développement personnel, les différences de salaires elles apparaissent moins justifiables à part le degré d'ancienneté». 
qui dérogent à l'exigence de proportionnalité - et donc de justice - entre les termes mis en rapport, sont un motif légitime de dénonciation de l'injustice. Ainsi bien des individus socialement peu avantagés explicitent, en toute impartialité et indépendamment de leur objectivité positionnelle, ce que serait, à leurs yeux, un «ordre social juste». Les récits de ces individus permettent, en outre, d'esquisser des représentations communes d'une société juste, du point de vue des individus les moins avantagés par la structure sociale.

\subsection{L'HYPOTHÈSE DE LA COHÉRENCE ARGUMENTATIVE}

L’orientation interprétative privilégiée par Axel Honneth s'explique par les présupposés encadrant sa réflexion et dont la validité s'avère empiriquement discutable. Son interprétation présuppose que l'implication émotionnelle du sujet agissant, lorsqu'il évalue des situations particulières, constituerait un obstacle majeur à toute cohérence argumentative. L'hypothèse d'une cohérence argumentative de la conscience morale quotidienne a émergé dans l'éthique philosophique ainsi que dans la psychologie développementale de Kohlberg mais a été ultérieurement mise en cause, notamment par la théorie de la personnalité, de sorte qu'un «cas normal» de conscience morale cohérente a été récusé (voir Döbert, 1980). Bien que la cohérence argumentative déserte, dans les entretiens recueillis, certaines réflexions sur la justice, elle est attestée par l'essentiel d'entre eux, quelle que soit la position du locuteur dans l'espace social. Plusieurs raisons expliquent et fondent cette cohérence.

En premier lieu, lorsqu'ils sont interrogés sur les inégalités et les injustices de la société française, les individus, y compris placés dans des positions de désavantage social, expriment des représentations morales, articulées de façon cohérente voire systématique, et esquissent des interprétations globales du fonctionnement social. Pressés par l'exigence de donner sens à leur existence et à leur position dans l'ordre social, ils se voient contraints d'essayer de comprendre ou d'interpréter le fonctionnement de cet ordre. Cette exigence explique le crédit conféré à la fiction légitimante du mérite (Dubet, 2004) ou celui accordé à la transmission du patrimoine et à sa naturalité supposée. De même, l'impli- 
cation émotionnelle associée à des situations d'injustice subies ou non n'est pas moins forte, que les individus soient ou non avantagés socialement.

En deuxième lieu, l'implication émotionnelle n'interdit aucunement que l'expression de sentiments d'injustice se formule en convoquant, implicitement ou explicitement, des références normatives. Enfin, récuser cette cohérence argumentative revient notamment à remettre en question la capacité réflexive individuelle à formuler des jugements en termes d'équilibre réfléchi, lorsque l'agent se trouve personnellement soumis à une situation d'injustice et à des formes de désavantage social. Or l'analyse des récits atteste de cette capacité, quelle que soit la situation individuelle assumée par le locuteur. Ainsi Juliette, salariée du privé en CDI et diplômée du supérieur, déplore, pour une part, l'insuffisante reconnaissance de ses compétences, au sein de son entreprise, mais considère pourtant que :

[...] pour ce que je fais, je trouve que ma rémunération elle est très bien. C’est un travail qui ne demande pas de capacités. C'est un travail où les gens qui travaillent avec moi, ils ont arrêté l'école en ze. En plus, je gagne i 350 euros net, donc pour quelque chose qui ne demande rien. C'est la même chose qu'un prof. Je fais trente-cinq heures, pas une minute de plus, pas une minute de moins. Non, franchement... [...] Je dis ça aussi parce que je n’ai pas de loyer à payer. $[. .$.$] Avec un loyer, I zoo euros ce n'est pas assez...$

Le jugement d'injustice n’est donc pas systématiquement biaisé par le point de vue personnel et égocentré.

\section{LÉGITIMATION, DÉNONCIATION, EXPLICATION Structures du discours vs. dichotomie entre groupes sociaux}

L’ultime projection réalisée par Axel Honneth sur la partition dichotomique entre groupes sociaux, dominants et dominés - i.e. sa troisième thèse dans le domaine (3) -, concerne non plus les attitudes normatives mais les registres du discours, opposant la justification ou la légitimation à la dénonciation. 
De façon manifeste, les «contraintes sociales de légitimation» pèsent sur le discours et l'argumentaire des individus socialement les plus avantagés, sur ceux dont les revenus sont les plus confortables et / ou occupant des positions de recruteur ou d'employeur. Selon Axel Honneth, ces individus se voient obligés de justifier, pour eux-mêmes comme pour les autres membres de la société, l'ordre social qui les privilégie, à la différence de ceux qu'il dessert. Cependant l'absence de discours de légitimation, du côté socialement le moins avantagé, peut s'expliquer par d'autres facteurs tels que le faible niveau d'éducation et le nombre plus restreint d’informations auquel les individus ont été exposés durant leur formation, par l'intériorisation de la fatalité face au déploiement des mécanismes sociaux et face à l'inévitabilité de la reproduction sociale, éventuellement par le fait de ne pas imaginer d'autres alternatives à l'ordre social existant ni de croire à leur avènement.

Dans le contexte sociohistorique des années 1980 auquel Axel Honneth fait référence, les «classes inférieures», en particulier les professions d'exécution - comme c'est encore aujourd'hui le cas - échappaient à ces exigences de justification. Y sont également soustraites nombre de professions où s'illustrent des phénomènes de domination sociale ou des situations d’asymétrie de pouvoir, en l'occurrence dans le monde de l'édition, du spectacle, de l'aide sociale (avec les travailleurs sociaux), de la santé (pour les infirmières et les aides-soignantes) voire de l'éducation (dans le cas des instituteurs). Les formes de la domination professionnelle, affectant le statut des salariés, subissant des conditions de travail dégradées (tels les précaires du spectacle ou les salariés du privé embauchés sur des contrats iniques), ne préjugent en rien de la nature des fonctions occupées ni des capacités requises pour les accomplir. Les individus tributaires de ces statuts ou occupant ces positions peuvent avoir un niveau de diplôme supérieur au baccalauréat et se trouver ainsi davantage préparés - du fait de l'information à laquelle ils ont été confrontés - à prendre, sur l'organisation sociale, une vue compréhensive qui leur permet d’en saisir les mécanismes. Cette appréhension fine de la réalité sociale et de son fonctionnement est en particulier présente, pour des raisons de proximité professionnelle, dans les métiers du social et de l'éducation. La contrainte de légitimation ne constitue donc pas l'unique motif expliquant la capacité individuelle à saisir l'imbrication des phénomènes sociaux 
et des phénomènes macroéconomiques. Le rôle des niveaux de diplôme et la confrontation à certaines réalités sociales revêtent une part explicative majeure.

En outre, la contrainte de légitimation se module distinctement selon les contextes. Elle se révèle d'autant plus forte, sur les individus les plus avantagés socialement, que pointe la question de la reproduction sociale ou que se trouve abordé le thème des inégalités de revenus et de la transmission du patrimoine (voir Galland et Lemel, 20II). L'intensité de la contrainte de légitimation varie donc à la fois selon les individus interrogés, selon les sphères de la justice considérées (comme la transmission du patrimoine), selon les orientations politiques individuelles et enfin selon les positions dans l'espace social mais d'une façon qui n'est nullement indépendante des catégories d'inégalités considérées. Ainsi cette contrainte pèse et traverse davantage le discours des individus privilégiés par l'ordre social, votant à droite plutôt qu'à gauche. La cohérence des représentations morales, rendue perceptible par la congruence du discours de la légitimation ou de la dénonciation des inégalités avec le positionnement politique contribue, une nouvelle fois, à ébranler l'interprétation honnethienne de la spécificité des représentations de l'ordre social, selon les positions sociales.

Enfin, la dichotomie entre justification et condamnation est en outre réductrice car d'autres types de discours, lorsqu'il est question de faits sociaux, sont possibles, tels que l'explicitation et l'explication (susceptible de convoquer la démonstration), la description, la qualification normative (qui peut tendre, à certaines occasions, vers la critique ou la déploration), la dénonciation, la révolte. Dénoncer et combattre les différences inhérentes à une structure sociale ou, à l'inverse, les accepter, les revendiquer, voire les valoriser, font partie des registres possibles du discours qui accompagne des attitudes de légitimation et des positions critiques. Axel Honneth n’a pas proposé de traitement spécifique de ces registres intermédiaires entre la justification et la dénonciation que sont la description et l'explication, dont le potentiel normatif peut être jugé plus restreint que celui de la légitimation ou de la dénonciation, ces modalités du discours n'encourageant pas l’action ni la mobilisation des agents ${ }^{13}$.

I3 E. Renault (2010) évoque une autre piste, en suggérant que le registre de l'explicitation déploie des arguments que les individus développent pour eux-mêmes, et celui de la justification des arguments développés pour autrui. 


\section{CONCLUSION}

Les analyses proposées visaient donc à réévaluer les thèses honnethiennes concernant le rapport à l'injustice de groupes sociaux antagonistes, c'est-à-dire, en particulier, l'hypothèse d'une dichotomie stricte entre deux types de représentations de la justice sociale, celle de l'organisation des représentations, dans le domaine, en un système organisé de normes abstraites exclusivement caractéristique des groupes socialement avantagés et enfin le confinement des représentations de la justice des plus défavorisés, dans des revendications réactives ou dans l'expression brute de sentiments d'injustice. Dans la mesure où ces thèses ainsi que celles de B. Moore (1978) s'appuient sur des données sociologiques datant des années 1970, une évolution des représentations du juste des groupes sociaux considérés a pu être mise en évidence. Nous avons ainsi montré que la morale sociale des individus et des groupes opprimés ne traduit pas seulement «une sensibilité exacerbée aux atteintes contre des revendications morales jugées légitimes » (Honneth, 2006, p. 208) mais également des représentations abstraites d'un ordre moral général. Leurs sentiments d'injustice n'expriment pas seulement en creux des exigences de justice, des besoins et des idées du bonheur mais se cristallisent aussi en projets explicites, fondés sur des principes axiologiques précis, d’une société juste. L’attitude normative des individus bénéficiant le moins de la structure sociale se porte au-delà de l'expression brute et anté-discursive de sentiments d'injustice. Elle ne trahit aucune «infériorité cognitive des classes dominées » (ibid., p 209). L'étude des représentations et des jugements sur la justice sociale et les inégalités a en outre permis de préciser certaines des modalités de l'articulation entre expression de l'injustice et jugements normatifs de justice. Cet examen de discours, recueillis dans le cadre d'une enquête empirique qualitative récente, a ainsi mis en lumière la spécificité des registres de l'énonciation, en matière de justice et d'inégalités, ainsi que l'écueil d'une projection de ces derniers sur des groupes sociaux distincts. 


\section{BIBLIOGRAPHIE}

BOURDIEU P., 2000, Les Structures sociales de l'économie, Paris, Seuil.

CAILLE A., LAZZERRI C. (dir.), 2009, La Reconnaissance aujourd'hui, Paris, CNRS Éditions.

DERANTY J.-P., 2009, Beyond Communication. A Critical Study of Axel Honneth's Social Philosophy, Boston et Leiden, Brill.

-, 2009, «La reconnaissance hégélienne et ses enjeux pour la philosophie sociale et politique contemporaine», Politique et Sociétés, 28-3, p.45-74.

-, 2011, «Travail et expérience de la domination dans le néolibéralisme contemporain », Actuel Marx, 49-I, p. 73-89.

DUBET F., 2004, L'École des chances, Paris, Seuil.

FISCHBACH F., 20I0, Manifeste pour une philosophie sociale, Paris, La Découverte.

GALLAND O., LEMEL Y, 20II, «Inégalités acceptables et inacceptables》, in M. Forsé et $O$. Galland (dir.), Les Français face aux inégalités et à la justice sociale, Paris, Colin, p. I08-117.

GUIBET LAFAYE C., 2012, Le Juste et l'Inacceptable. Les sentiments d'injustice contemporains et leurs raisons, Paris, PUPS.

HONNETH A., 198I, «Moralbewusstein und soziale Klassenherrschaft. Einige Schwierigkeiten in der Analyse möglicher normativer Handlungspotentiale», in Leviathan Zeitschrift für Sozialwissenschaft, 9-(3-4), p. 556-570.

-, 2000 (1992), La Lutte pour la reconnaissance, Paris, Cerf.

-, 2006 (2000), La Société du mépris, Paris, La Découverte.

-, 2007 (2005), La Réification: petit traité de théorie critique, Paris, Gallimard.

-, 2008, Les Pathologies de la liberté, Paris, La Découverte.

ISER M., 2008, Emporung und Fortschritt. Grundlagen einer Kritischen Theorie der Gesellschaft, Frankfurt/New York, Campus.

KANT E., 1990 (1790), Kritik der Urteilskraft, Felix Meiner Verlag, Hambourg; tr. fr. A. Renaut, Critique de la faculté de juger, 1995, Paris, Aubier.

-, 1986 (1795), Projet de paix perpétuelle, tr. fr. in Fuvres complètes, Paris, Gallimard, t. III. 
LINHART D., 20II, «De la domination et de son déni », Actuel Marx, 49-I, p.90-I03.

MAYER N., 2003, «Que reste-t-il du vote de classe?», Lien social et Politiques, 2003, 49, p. I0I-III.

MENGER P.-M., 20II (2005), Les Intermittents du spectacle. Sociologie du travail flexible, Paris, EHESS, coll. «Cas de figure».

MOORE B., 1978, Injustice. The Social Basis of Obedience and Revolt, New York, White Plains.

PARSONS T., 1967 (1963), «On the Concept of Political Power», Sociological Theory and Modern Society, New York, The Free Press, p. 297-354.

POGGE T., 1988, «Kant's Theory of Justice», Kant-Studien, 79-4, p. 407-433.

RAINER D., 1980, «Normen, Einsichten und Handeln», Kursbuch, 60, p. 43-59.

RAINER F. (dir.), 2009, Sozialphilosophie und Kritik, Frankfurt, Suhrkamp.

ULRICH K., 2009, Die Sozialphilosophie von Axel Honneth, Munich, Grin.

RENAULT E., 2004, L'Expérience de l'injustice, Paris, La Découverte.

-, «Injustice et reconnaissance », communication proposée le 10 mai 2010, dans le séminaire «Dilemmes moraux pratiques et justice sociale», Paris, EHESS.

-, SINTOMER Y. (dir.), 2003, Où en est la théorie critique?, Paris, La Découverte.

VAN DEN BRINK B., OWEN D., 2007, Recognition and Power: Axel Honneth and the Tradition of Critical Social Theory, Cambridge, Cambridge University Press.

\section{ANNEXE I : LISTE DES PERSONNES INTERROGÉES}

Anne, 27 ans, salariée du privé en CDI, documentaliste-rédactrice.

August, 56 ans, ouvrier qualifié.

Béatrice, 4I ans, enseignante de musique.

Brigitte, 50 ans, cadre supérieur titulaire du public, conseillère d'orientation.

Catherine, 42 ans, cadre supérieur, profession libérale.

Charles, 28 ans, ingénieur du son, intermittent du spectacle.

Charlotte, 27 ans, assistante sociale en CDI.

Daniel, 30 ans, journaliste à son compte, pigiste.

Dominique, 5I ans, chômeuse antérieurement conseillère en insertion professionnelle.

Estelle, 5 I ans, profession intermédiaire du privé en CDI dans l'agro-alimentaire. 
Fabien, 28 ans, chef d'entreprise, auto-entrepreneur.

Fabienne, 58 ans, directrice de librairie.

Félix, 57 ans, chef d'entreprise, auto-entrepreneur.

François, 34 ans, travailleur social, salarié du privé en CDI.

Gaëlle, 63 ans, retraitée, anciennement institutrice et directrice d'école.

Habib, 56 ans, conseiller principal d'éducation (CPE), cadre moyen titulaire du public.

Harold, 56 ans, statisticien du Pôle Emploi.

Henry, 52 ans, profession intermédiaire du privé en CDI de chantier (CDIC).

Jacques, 50 ans, chômeur antérieurement infographiste dans le privé.

Jannick, 57 ans, ouvrier qualifié.

Jean-Baptiste, 44 ans, cadre supérieur du privé.

Jean-Yves, 64 ans, ouvrier qualifié.

Jeanne, 29 ans, profession intermédiaire du privé en CDI.

Jessica, 27 ans, auto-entrepreneur.

Julie R., 27 ans, profession intermédiaire du privé en CDI.

Juliette, 26 ans, salariée du privé en CDD.

Karine, 33 ans, profession intermédiaire du privé aujourd'hui au chômage et en formation professionnelle.

Laetitia, 40 ans, artiste à son compte.

Laurence, 38 ans, ouvrière qualifiée.

Madeline, 56 ans, conteuse intermittente du spectacle.

Marcel, 39 ans, ouvrier qualifié.

Marie G., 38 ans, agent d'accueil CROUS.

Marie L., 44 ans, profession intermédiaire en congé parental.

Marie-Claude, 62 ans, retraitée, travaillant à mi-temps, anciennement commerçante.

Marie-Pierre, 65 ans, femme au foyer.

Marine, 20 ans, étudiante aide-soignante, employée en CDD dans le privé.

Max, 73 ans, retraité anciennement artisan commerçant et chef d'entreprise.

Michel, 46 ans, intermittent du spectacle.

Mouna, 60 ans, femme au foyer.

Pascal, 38 ans, ouvrier qualifié.

Patrick, 59 ans, pharmacien.

Pierre C., 52 ans, cadre supérieur du privé.

Raïssa, 28 ans, professeur des écoles.

Richard, 40 ans, cadre supérieur dirigeant de PME.

Sarah, 75 ans, journaliste et psychologue en activité.

Sébastien, 40 ans, professeur des écoles.

Thibault, 44 ans, ouvrier qualifié.

Thierry, 24 ans, conducteur de trains.

Thomas, 37 ans, cadre supérieur du privé.

Vincent, 29 ans, éducateur.

Vivianne, 28 ans, psychomotricienne. 


\section{ANNEXE 2: EXTRAIT DES QUESTIONS POSÉES AUX ENQUÊTÉS}

- «Il y a dans la société française différentes formes d’inégalités. Quelles sont selon vous les grandes inégalités qui caractérisent aujourd'hui la société française?

Est-ce que certaines inégalités vous paraissent plus acceptables que d'autres?

Inévitables? »

- «Tout bien considéré, dans l'ensemble, diriez-vous que la société française est plutôt juste ou plutôt injuste?»

- «J’aimerais revenir sur les différentes formes d’inégalités car nous ne les avons pas toutes abordées. On peut par exemple penser aux inégalités suivantes: les inégalités de patrimoine, de logement, scolaires; les inégalités face au chômage et aux emplois précaires, aux conditions de travail, entre les hommes et les femmes, liées à l'origine ethnique, face à la santé et aux soins, entre les jeunes et les plus âgés, face à l'insécurité, d'exposition aux risques technologiques, industriels ou scientifiques (nucléaire, OGM, etc.). Y en a-t-il certaines qui vous semblent particulièrement fortes en France aujourd'hui?»

- «Pensez-vous que l'État devrait davantage intervenir pour réduire les inégalités? Quelles devraient être ses priorités, selon vous?

S'agissant de la protection sociale, certains disent qu'il faut aider tout le monde et d’autres pensent au contraire qu'il ne faut pas aider ceux qui ne font pas d'effort? Qu'en pensez-vous?

Prenons les chômeurs, par exemple, certains disent que ceux qui ne recherchent pas activement un emploi devraient perdre leurs indemnités. Qu'en pensez-vous?»

- «Voici trois sociétés imaginaires ayant chacune trois catégories sociales.

(Enquêteur : montrer carton B + faire remarquer ce qui figure en italique sur le carton à savoir : il y a moins d'inégalité et de richesse dans la société $\mathrm{B}$ que dans la société $\mathrm{C}$ et encore moins dans la société $A$ que dans la société $\mathrm{B}$. Si l'enquêté soulève la question, préciser que le prix des biens est identique dans les trois sociétés)

Sans tenir compte de votre situation personnelle (puisqu'il s'agit de sociétés purement imaginaires), dans quelle société préféreriez-vous vivre? La société A, B ou C?

Et, globalement, quelle société vous paraît la plus juste? La société A, B ou C ? Pourquoi ?» 